\title{
Thrombolysis for Prevention of Cerebral No-Reflow After Cardiopulmonary Resuscitation
}

\author{
K.-A. Hossmann¹, V. Hossmann², B. W. Böttiger ${ }^{3}$ \\ ${ }^{1}$ Max Planck Institute for Neurological Research, Cologne, \\ ${ }^{2}$ Department of Internal Medicine, Academic Hospital Cologne-Porz, \\ ${ }^{3}$ Department of Anesthesiology and Postoperative Intensive Care Medicine, University of Cologne
}

\begin{abstract}
Thrombolytic treatment during cardiopulmonary resuscitation is thought to reverse the cerebral no-reflow phenomenon which is widely considered to limit neurological recovery after prolonged cardio-circulatory arrest. However, the recent multicenter randomized double-blind TROICA (Thrombolysis in Cardiac Arrest) trial revealed that patients with witnessed out-of-hospital cardiac arrest did not experience an improvement of neurological outcome when treated with the thrombolytic agent tenecteplase [1]. This raises the question of the importance of coagulation disturbances in the pathophysiology of no-reflow and its reversal by thrombolytic interventions. This article provides an overview of the experimental literature on this subject. Key words: cardiac arrest, no-reflow phenomenon, neurological recovery, disseminated intravascular coagulation, thrombolysis.
\end{abstract}

Introduction

Over the past decades considerable methodological and logistic advances in out-of hospital resuscitation have been achieved but the overall prognosis of cardiac arrest is still poor. In search of new therapeutic options, attention has been directed to the use of fibrinolytic drugs applied during cardiopulmonary resuscitation [2]. There are two major arguments in support of this concept. One is the reversal of macrovascular occlusions associated with myocardial infarction and pulmonary embolism which account for approximately $70 \%$ of all cardiac arrest cases [3]. The other is amelioration of microcirculatory obstructions resulting from disseminated coagulopathy which develops after circulatory arrest due to an imbalance between coagulation and fibrinolysis [4]. The latter is of particular importance for the brain because it may contribute to the no-reflow phenomenon which is thought to limit neurological recovery after cardiac arrest exceeding 8-10 min duration [5].

Many case reports and several small-number therapeutic studies using urokinase, streptokinase, alteplase, rtPA or the recently developed tPA isomer tenecteplase are in support of this concept [6-16]. Interestingly, these reports included not only a high rate of return of spontaneous circulation (ROSC) but also a surprisingly high number of neurologically intact survivors, even after prolonged cardio-pulmonary resuscitation (CPR) [17]. Several small prospective and uncontrolled studies suggested a beneficial effect of thrombolytic drugs administered during CPR in patients suffering from acute massive pulmonary embolism [18], acute myocardial infarction [19] and in patients who had been unresponsive to conventional resuscitation efforts [20]. In a prospective study in out-

Адрес для корреспонденции (Correspondence Address):

Hossmann Konstantin-Alexander

E-mail: hossmann@nf.mpg.de of-hospital cardiac arrest patients, we have compared patients treated with heparin and alteplase and patients with standard therapy, if ROSC was not achieved within 15 minutes of conventional CPR [9]. Patients treated with alteplase were more likely to achieve ROSC and hospital admission compared to controls. This were supported by a large-scale retrospective case-control study in 324 out-ofhospital cardiac arrest patients demonstrating a hospital discharge rate of $25 \%$ vs. $15 \%$ [21].

An inherent risk of thrombolytic treatment is bleeding. A meta-analysis of thrombolysis during CPR did not reveal an increase of significant bleedings [22] but in view of the potential risk of such complications, and as several studies did not confirm improvement of outcome [23-25], a multicenter randomized double-blind, placebo-controlled trial (The Thrombolysis in Cardiac Arrest (TROICA) study) has been carried out $[1,26]$. The TROICA trial is a European multicenter trial with 1,050 patients suffering from witnessed out-of-hospital cardiac arrest of presumed cardiac origin. Patients were randomized to a weightadjusted dose of tenecteplase or placebo during CPR, without any concomitant anticoagulant therapy. Interestingly, there were no differences between tenecteplase and placebo in 30-day survival (15\% vs. 17\%), ROSC and hospital admission. There were also no significant differences in cerebral outcome, but there was a trend towards a higher number of patients with good cerebral recovery, i.e. cerebral performance categories 1 or 2, in the tenecteplase group (63\% vs. $56 \%)$ [1].

All this raises the question to what extent cerebral no-reflow affects neurological recovery after cardiac arrest, and if it does so, whether thrombolysis can be expected to prevent this complication. In this communication experimental data dealing with this question are reviewed.

\section{No-reflow of the brain after cardiac arrest}

The no-reflow phenomenon was first described by Ames et al. who studied the recirculation of the brain after 


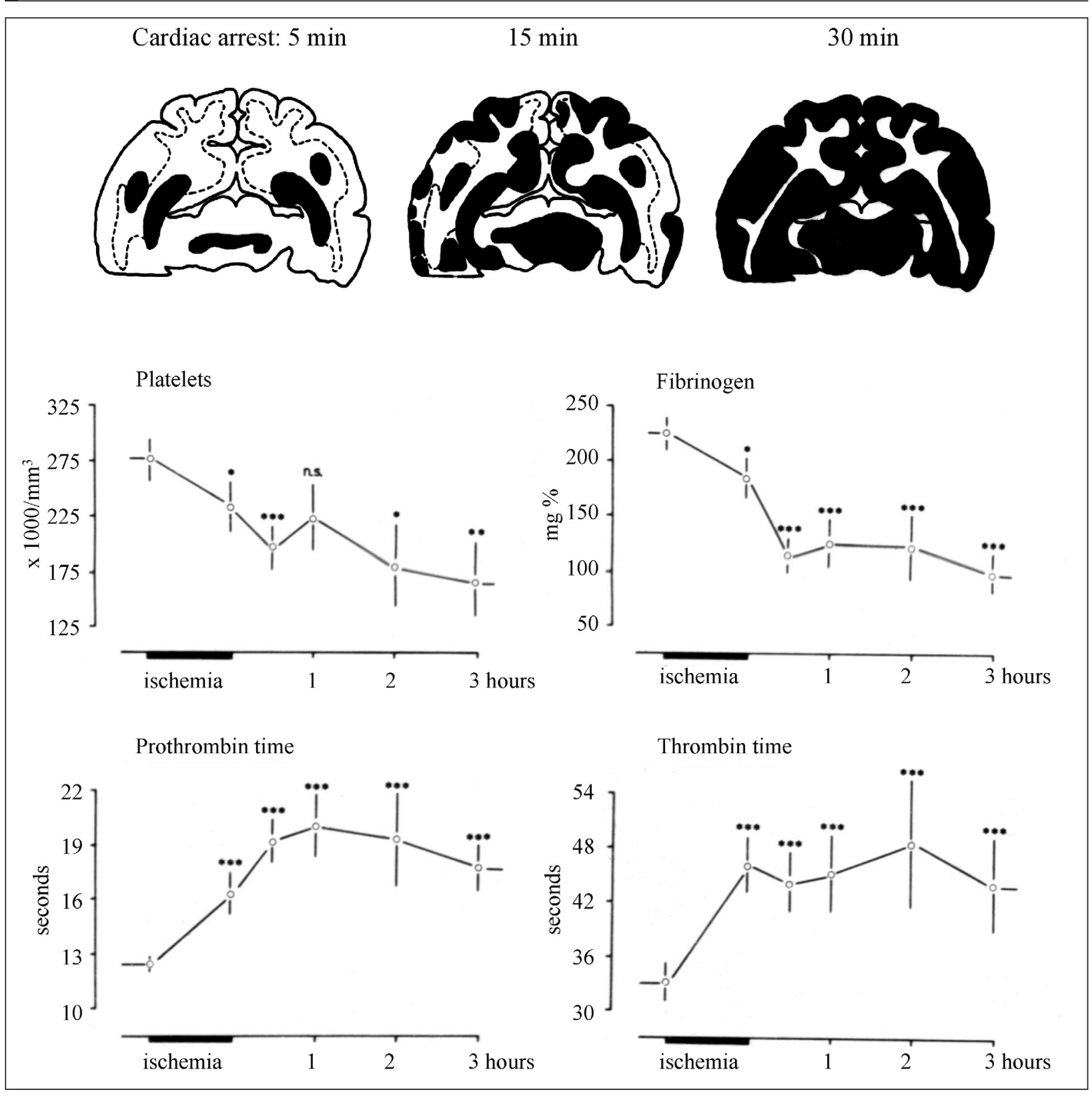

Fig. 1: Pattern of no-reflow in cat brain after successful resuscitation from 5, 15 and 30 min cardiac arrest. No-reflow (black) areas were visualized $30 \mathrm{~min}$ after return of spontaneous cardiac function by labeling circulating blood with FITC-albumin. Modified from [32].

increasing durations of global cerebrocirculatory arrest by staining the blood with carbon black prior to reperfusion [27]. Cerebrocirculatory arrest was induced in rabbits by inflating a pneumatic cuff placed around the neck of the animals to above arterial blood pressure, and reperfusion was initiated by releasing the pressure from the cuff. The investigation was prompted by the observation that in the isolated retina evoked potentials fully recovered in vitro after up to $20 \mathrm{~min}$ glucose and oxygen deprivation at $37^{\circ} \mathrm{C}$ [28] whereas normothermic circulatory arrest in vivo led to irreversible brain injury within less than 8-10 min [29]. Assuming that the anoxic sensitivity of retinal neurons is similar to that of the brain, Ames and his colleagues hypothesized that the difference must be related to the vascular system and that reoxygenation in vivo by the circu- lating blood is less efficient than the restoration of oxygen supply in vitro. The results confirmed that with increasing duration of ischemia in vivo an increasing part of the brain was not recirculated with blood: after $7.5 \mathrm{~min}$ circulatory arrest up to $50 \%$ and after 15 min up to $95 \%$ of brain volume suffered no-reflow [27]. As the shortest ischemia time at which substantial no-reflow became prominent, i. e. 7.5 min, came close to the previously established limits of brain tolerance to circulatory arrest, the authors concluded, that the high vulnerability of the brain to ischemia might be due to secondary post-ischemic hemodynamic rather than to primary ischemic cellular dysfunction.

Further studies into the mechanisms of no-reflow revealed that that this disturbance is a microvascular disorder caused by the combination of endothelial and perivas- 
cular glial swelling, an increase in blood viscosity due to intravascular coagulation, hemoconcentration and aggregation of platelets at low shear, and the formation of endothelial blebs derived from protrusions of endothelial cells (for review see [5, 30]). The combined effect of these alterations is an increase in microvascular resistance which can only be overcome by an increase in post-ischemic reperfusion pressure. Using the pneumatic cuff method for the production of cerebrocirculatory arrest, the reperfusion pressure required to prevent no-reflow amounts to about $60 \mathrm{mmHg}$ after $10 \mathrm{~min}$ ischemia, $80 \mathrm{mmHg}$ after $15 \mathrm{~min}$ ischemia and over $160 \mathrm{mmHg}$ after $30 \mathrm{~min}$ ischemia [31].

This relationship is the reason that no-reflow is of particular concern for cardiac arrest where conventional resuscitation procedures may not be able to built up the blood pressure required for reperfusion, particularly after longer durations of ischemia. Several experimental studies are in support of this assumption. Fischer et al. induced ventricular fibrillation in cats, using internal bipolar electrical stimulation [32]. After 5, 15 or 30 min cardiac arrest, advanced cardiopulmonary resuscitation was carried out by closed-chest cardiac massage in combination with mechanical ventilation with $100 \%$ oxygen and the intravenous infusion of $0.2 \mathrm{mg} / \mathrm{kg}$ epinephrine and $2 \mathrm{mEq} / \mathrm{kg}$ sodium bicarbonate. Electrical defibrillation was attempted 4 min later and - if unsuccessful - repeated after additional 2-3 min periods of cardiac massage and $0.1 \mathrm{mg} / \mathrm{kg}$ epinephrine injections. Reperfusion of the brain was visualized by labeling the circulating blood with fluoresceine isothiocyanate (FITC)-albumin, and areas of no-reflow were defined as absence of microvascular filling. The filling defects were identified by fluorescence microscopy at 8 standard coronal levels of forebrain, and expressed as percent of total sectional area. During cardiac massage noreflow affected $21 \%, 42 \%$ and $70 \%$ of forebrain after 5,15 and 30 min cardiac arrest, respectively. Following successful resuscitation of the heart and 30 min spontaneous recirculation at mean arterial pressure of about $80 \mathrm{mmHg}$, noreflow resolved only after $5 \mathrm{~min}$ cardiac arrest to $7 \%$ $(p<0.05)$ but persisted in $30 \%$ and $65 \%$ of forebrain after 15 and 30 min cardiac arrest, respectively (n. s.) The topical distribution of no-reflow areas was multifocal and exhibited a time-dependent centripetal expansion with increasing ischemia time (Fig. 1). After 5 min cardiac arrest it mainly affected hippocampus and multiple small areas in basal ganglia, after $15 \mathrm{~min}$ it expanded into cerebral cortex, and after $30 \mathrm{~min}$ all parts of the brain were involved.

The importance of the initial post-ischemic reperfusion pressure for the manifestation of no-reflow was also documented by Böttiger et al. [33] who induced cardiac arrest in rats by electrical ventricular fibrillation and compared resuscitation by basic life support, i.e. mechanical ventilation and external cardiac compression, with advanced resuscitation procedures, in which epinephrine and sodium bicarbonate were applied additionally to accelerate the return of functionally efficient reperfusion pressure. During basic life support mean arterial blood pressure amounted to $27 \mathrm{mmHg}$, and during advanced resuscitation procedures to about $65 \mathrm{mmHg}$. $12 \mathrm{~min}$ cardiac arrest followed by $5 \mathrm{~min}$ basic life support before advanced resuscitation was started resulted in no-reflow in $6.9 \%$ of brain volume, whereas cardiac arrest of 17 min followed immediately by advanced resuscitation reduced no-reflow to $0.7 \% \quad(p<0.05)$. Resuscitation at high perfusion pressure, therefore, appears to be beneficial even if ischemia time is prolonged.

This explains occasional reports on successful brain resuscitation after normothermic cardiac arrest of up to 30 min when reperfusion pressure was raised by either vigorous intrathoracic cardiac massage [34], or by using mechanical extracorporeal circulation $[35,36]$. Brain reperfusion at elevated blood pressure level was also carried out by Seo et al. using the so-called two-stage resuscitation protocol [37]. In this experiment cats were submitted to 30 min KCl-induced cardioplegia, followed by closed chest cardiac massage. During cardiopulmonary resuscitation the brain was disconnected from the general circulation by inflating a pneumatic cuff around the animal's neck until spontaneous circulation with systolic blood pressure over $100 \mathrm{mmHg}$ returned. Although this procedure prolonged the ischemia time of the brain from $30 \mathrm{~min}$ to as long as 1 hour, regional energy metabolism returned to normal in 6 out of 13 animals, indicating not only successful prevention of no-reflow but also functional recovery [37]. Finally, reflow at high reperfusion pressure was extensively investigated in a model of prolonged selective brain ischemia. In this model brain circulation was completely interrupted by intrathoracic occlusion of the innominate, subclavian and internal mammary arteries in combination with pharmacologically induced hypotension. As the heart was not affected by this procedure, systolic arterial blood pressure could be raised pharmacologically well over $200 \mathrm{~mm} \mathrm{Hg}$ prior to vessel release, resulting in instantaneous reperfusion and the development of pronounced hyperemia. Prevention of no-reflow was associated with successful metabolic, electrophysiological and even neurological brain recovery and could be achieved without any further interventions after normothermic circulatory arrest of up to 1 hour [38]. The combined evidence of these data suggests that the high incidence of brain injury after much shorter periods of cardiac arrest is, in fact, due to cerebral no-reflow, and that prevention of no-reflow is a reasonable strategy to improve neurological outcome.

\section{Contribution of disseminated coagulopathy to no-reflow}

The proposition of the contribution of microvascular coagulation to the development of no-reflow is inferential and mainly based on the observation that cerebrocirculatory arrest induces disseminated intravascular coagulation (DIC) [4,39-43]. In our laboratory one hour selective brain ischemia induced pronounced consumption coagulopathy with dramatic decrease in the blood content of platelets and fibrinogen that was associated with reciprocal prolongation of coagulation times (Fig. 2) [44]. 


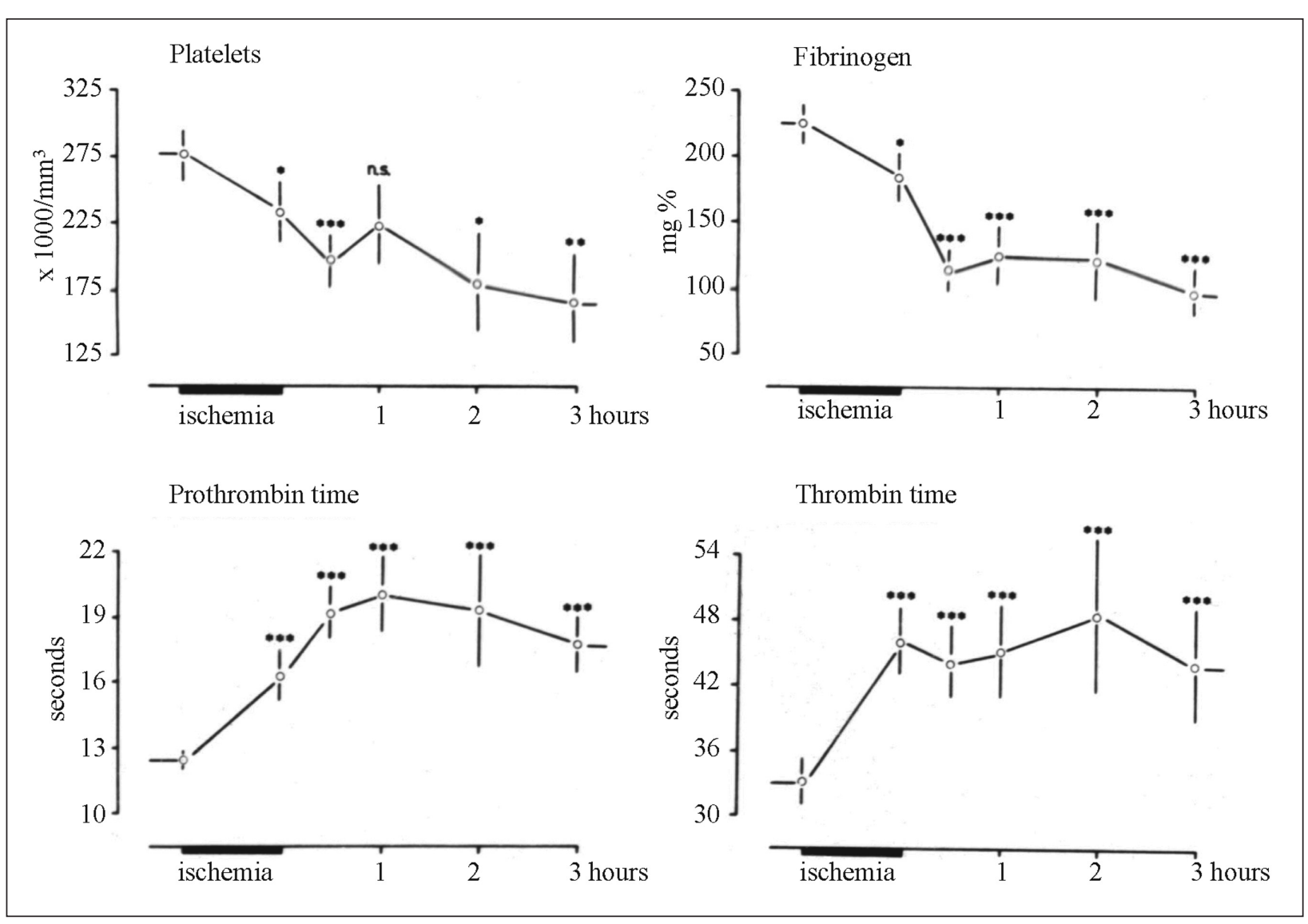

Fig. 2: Disseminated coagulopathy following 1 hour cerebro-circulatory arrest induced in cat by intrathoracal clamping of the brachiocephalic and subclavian arteries. Note progressive reduction of platelets and fibrinogen in the circulating blood associated with reciprocal lengthening of the clotting times. Modified from [44].

DIC is a multifactorial process triggered by ischemic/hypoxic damage of the endothelium, thereby exposing pathological levels of tissue factor. By inducing intrinsic and extrinsic activation of the coagulation cascade an uncontrolled generation of thrombin leads to systemic fibrin deposition in the non-capillary microvasculature. In addition platelets are activated, mainly via the thromboxane A2-pathway to form platelet aggregates, which further impair the microcirculation. There is also clear indication of a systemic inflammatory response as evidenced by activation of neutrophils. Interestingly, patients with acute coronary syndrome, who are at high risk of cardiac arrest, exhibit a pronounced hypercoagulable state already before the ischemic event, as demonstrated by increased levels of thrombin-antithrombin complex (TAT-complex) and of plasminogen activator inhibitor-1 (PAI-1). These changes reflect the increased turnover in thrombin, being inactivated by the formation of TAT-complexes, on the one hand, and by impaired fibrinolysis due to high concentration of PAI-1, on the other. The latter is released in excess from the damaged endothelial cells to inhibit cleavage of the fibrin clots by plasmin [45].

The only study which directly addressed the relationship between DIC and cerebral blood flow came from our laboratory and was carried out in cats submitted to 1 hour complete cerebrocirculatory arrest [46]. To detect microaggregates of platelets after reperfusion, platelets were labelled with $51 \mathrm{Cr}$. Blood volume was measured by injection of 125Iodine-albumin and the blood platelet count, together with serum fibrinogen, was determined in venous blood taken before ischemia and after reperfusion for $30 \mathrm{~min}, 2$ hours and 4 hours, respectively. The number of platelets entrapped in brain and peripheral organs was calculated by subtracting from the total tissue platelet count those contained in the circulating blood, as derived from the tissue blood content measured with 125I-albumin. Thirty minutes after the beginning of post-ischemic recirculation the number of entrapped platelets increased significantly in the whole brain, the highest number being found in the border zones and in the cerebellum. After 4 hours platelets were almost completely washed out of the brain but high numbers of aggregated platelets were detected in the kidney, the lung and the liver throughout the observation time. In the liver even a continuous increase was observed, possibly due to phagocytosis of platelets and platelet fragments by the reticulo-endothelial system (RES).

Interestingly, cerebral platelet aggregation did not result in ischemia but, quite contrary, was most pronounced at the time of post-ischemic hyperemia [46]. This is explained by the built-up of a high reperfusion pressure after selective cerebrocirculatory arrest which led to the restoration of blood circulation to most parts of cerebral microcirculation and which is in line with the observation that cerebral no-reflow can be prevented by raising blood 
reperfusion pressure above normal [31]. After cardiac arrest the situation is presumably different because during cardiac resuscitation reperfusion pressure is distinctly below normal. To the best of our knowledge experimental data are not available to document the contribution of intravascular coagulation to no-reflow under this circumstance, but even in the absence of such a direct involvement, intravascular coagulation may be detrimental. As platelets accumulate massively in peripheral organs, notably in the lung, the kidney and the liver, multi-organ failure may develop which might be of similar significance for the functional outcome of post-cardiac arrest brain resuscitation as the direct involvement in post-ischemic reperfusion. Prevention of disseminated coagulation may, therefore, be of considerable therapeutic interest irrespective of its putative role in the pathophysiology of no-reflow.

\section{Thrombolytic treatment of no-reflow}

An important motivation for thrombolysis during cardiac resuscitation is prevention of cerebral no-reflow but the first attempts to improve neurological outcome by anticoagulation were made well before the no-reflow phenomenon had been discovered.

Already in the middle of the last century, Crowell demonstrated beneficial effects of heparin treatment before cardiac arrest. Only very few dogs survived without heparin pretreatment, while the survival rate was $16 \%$ and $67 \%$, respectively, when a dose of $2 \mathrm{mg}$ and $5 \mathrm{mg}$ per $\mathrm{kg}$ body weight of heparin was given [47]. Crowell also demonstrated beneficial effects of pretreatment with thrombolytic agents. In the control group, 14 of 15 animals died after $15 \mathrm{~min}$ of cardiac arrest [48]. The surviving animal suffered from severe neurological damage. In contrast, only 2 of 14 animals died if streptokinase had been administered before cardiac arrest, and almost all neurological deficits in this group disappeared within 2 months. Some years later, Lin demonstrated that the administration of streptokinase combined with dextran reduces the duration of a flat line EEG and improves cerebral blood flow after cardiac arrest in dogs [49]. Safar and his group observed an improved neurological recovery in dogs receiving heparin, dextran and hypertensive reperfusion following cardiac arrest [50]. In accordance with these findings, a postmortem study in humans revealed intravascular fibrin formation in pulmonary and renal microvessels following resuscitation from cardiocirculatory arrest, but not in patients who died without CPR [51]. These data suggest that there is indeed an imbalance in hemostasis during reperfusion after cardiac arrest that can lead to intravascular fibrin generation and microthrombosis in the microcirculation of all organs including the brain.

Experimental evidence that fibrinolysis reduces noreflow has been provided only in one animal investigation (Table) [52]. In this study cats were submitted to $15 \mathrm{~min}$ ventricular fibrillation, followed by advanced cardiopulmonary resuscitation with or without the additional application of rtPA and heparin. Cardiac arrest was induced by
Thrombolytic treatment of no-reflow after 15 min cardiac arrest

\begin{tabular}{lcc}
\hline Brain region & Untreated & Treated \\
\hline Total forebrain & $28 \pm 13 \%$ & $7 \pm 6 \% *$ \\
Cerebral cortex & $27 \pm 14 \%$ & $8 \pm 1 \% *$ \\
Basal Ganglia & $63 \pm 26 \%$ & $15 \pm 20 \% *$ \\
Brainstem & $26 \pm 26 \%$ & $2 \pm 3 \% *$ \\
\hline
\end{tabular}

Values are cross-sectional areas $( \pm$ SEM) of no-reflow in percent of total brain, ${ }^{*}-p<0.05$. Data taken from [52].

electrical fibrillation and cardiopulmonary resuscitation by extrathoracal cardiac massage in combination with repeated injections of epinephrine and sodium bicarbonate. 30 min after the onset of cardiac resuscitation microvascular perfusion was assessed by staining the circulating blood with fluoresceine isothiocyanate (FITC)-albumin, and noreflow was identified by the absence of microvascular filling. Thrombolysis significantly reduced the extent of noreflow from $28 \%$ to $7 \%$ of brain volume, reflecting the substantial reversal of microthrombotic occlusions. Obviously, this finding provides a plausible explanation for thrombolysis-induced improvement of neurological recovery under experimental $[48,49]$ and clinical conditions [8, 14, 15, 53]. However, blood reperfusion may also improve due to other rtPA-induced effects such as the improvement of inotropic heart function [52] or the change in blood viscosity [54]. Thrombolysis is, therefore, not the only strategy for improving post-ischemic recirculation and should be evaluated in comparison to other, possibly less hazardous interventions.

\section{Conclusions}

In the TROICA trial the fibrinolytic agent tenecteplase, without concomitant anticoagulatory intervention, did not significantly improve cerebral outcome or the overall survival rate after out-of-hospital cardiac arrest. However, this does not necessarily refute the concept of thrombolytic amelioration of cerebral no-reflow because the postulated neurological improvement may show up only when cerebral no-reflow becomes the limiting factor for brain resuscitation, i. e., after circulatory arrest of longer than 8-10 minutes. In the light of a trend towards better overall neurological recovery in the tenecteplase group of the TROICA trial, it would be of interest to re-evaluate the neurological recovery in subgroups of patients with different durations of ischemia.

It is also conceivable that the experimentally documented thrombolytic alleviation of no-reflow was not mediated by reversal of intravascular coagulation but by reduction of blood viscosity which is known to improve post-ischemic recirculation. If the latter is an additional mechanism, thrombolytic agents being less fibrin and more fibrinogen specific than tenecteplase may induce more pronounced effects, because fibrinogenolysis can be expected to further improve cerebral microcirculatory reperfusion. Further experimental studies are warranted to test this hypothesis. 


\section{Литература}

1. Böttiger B.W., Arntz H., Chamberlain D. et al. Thrombolysis during resuscitation for out-of-hospital cardiac arrest. N. Engl. J. Med. 2008; 359 (25): 2651.

2. Böttiger B.W. Thrombolysis during cardiopulmonary resuscitation. Fibrinolysis \& Proteolysis 1997; 11: 93-100.

3. Newman D., Greenwald I., Callaway C. Cardiac arrest and the role of thrombolytic agents. Ann. Emerg. Med. 2000; 35 (5): 472-480.

4. Böttiger B. W., MotschJ., Bohrer H. et al. Activation of blood coagulation after cardiac arrest is not balanced adequately by activation of endogenous fibrinolysis. Circulation 1995; 92 (9): 2572-2578.

5. Hossmann K.-A. Reperfusion of the brain after global ischemia: hemodynamic disturbances. Shock 1997; 8 (2): 95-101; discussion 102-103.

6. Aliyev F., Habeb M., Babalik E. et al. Thrombolysis with streptokinase during cardiopulmonary resuscitation: A single center experience and review of the literature. J. Thromb. Thrombolysis 2005; 20 (3): 169-173

7. Bozeman W. P., Kleiner D. M., Ferguson K. L. Empiric tenecteplase is associated with increased return of spontaneous circulation and short term survival in cardiac arrest patients unresponsive to standard interventions. Resuscitation 2006; 69 (3): 399-406

8. Lederer W., Lichtenberger C., Pechlaner C. et al. Long-term survival and neurological outcome of patients who received recombinant tissue plasminogen activator during out-of-hospital cardiac arrest. Resuscitation 2004; 61 (2): 123-129.

9. Böttiger B. W., Bode C., Kern S. et al. Efficacy and safety of thrombolytic therapy after initially unsuccessful cardiopulmonary resuscitation: a perspective clinical trial. Lancet 2001; 357 (9268): 1583-1585.

10. Soltesz S., Berg K., Molter G. Successful thrombolysis of a severe pulmonary embolism during cardiopulmonary resuscitation. Anaesthesist 1997; 46 (10): 890-894.

11. Kuisma M., Silfvast T., Voipio V., Malmstrom R. Prehospital thrombolytic treatment of massive pulmonary embolism with reteplase during cardiopulmonary resuscitation. Resuscitation 1998; 38 (1): 47-50.

12. Schulte-Sinkus D., Standl T. Successful resuscitation after bolus injection of tissue-type plasminogen activator in emergency admission. Anasthesiol. Intensivmed. Notfallmed. Schmerzther 1998; 33 (2): 124-128.

13. Grabner $C$., Wahl U., Reineke H. Successful cardiopulmonary resuscitation with a high-dosage bolus injection of rt-PA after fulminant pulmonary embolism. Anasthesiol. Intensivmed. Notfallmed. Schmerzther 2001; 36 (5): 306-308

14. Schreiber W., Gabriel D., Sterz F. et al. Thrombolytic therapy after cardiac arrest and its effect on neurological outcome. Resuscitation 2002 52 (1): 63-69

15. Li X., Fu Q. L., Jing X. L. et al. A meta-analysis of cardiopulmonary resuscitation with and without the administration of thrombolytic agents. Resuscitation 2006; 70 (1): 31-36.

16. Fatovich D., Dobb G., Clugston R. A pilot randomised trial of thrombolysis in cardiac arrest (The TICA trial). Resuscitation 2004; 61 (3): 309-313.

17. Padosch S. A., Motsch J., Böttiger B. W. Thrombolysis during cardiopulmonary resuscitation. Anaesthesist 2002; 51 (7): 516-532.

18. Kurkciyan I., Meron G., Sterz F. et al. Pulmonary embolism as cause of cardiac arrest: presentation and outcome. Arch. Intern. Med. 2000; 160 (10): 1529-1535.

19. Gramann J., Lange-Braun P., Bodemann T. et al. Der Einsatz von Thrombolytika in der Reanimation als Ultima ratio zur Überwindung des Herztodes. Intensiv und Notfallbehandlung 1991; 16: 134-137.

20. Kleiner D., Ferguson K., King K. et al. Empiric tenecteplase use in cardiac arrest refractory to standard advanced cardiac life support interventions. Circulation 2003; 108: 318-319.

21. Lederer $W$., Lichtenberger $C$., Pechlaner $C$. et al. Recombinant tissue plasminogen activator during cardiopulmonary resuscitation in 108 patients with out-of-hospital cardiac arrest. Resuscitation 2001; 50 (1): 71-76.

22. Spohr F., Böttiger B.W. Safety of thrombolysis during cardiopulmonary resuscitation. Drug Saf. 2003; 26 (6): 367-379.

23. Abu-Laban R. B., Christenson J. M., Innes G. D. Effects of thrombolysis during out-of-hospital cardiopulmonary resuscitation. Am. J. Cardiol. 2006; 98 (3): 426-427.

24. Stadlbauer K. H., Krismer A. C., Arntz H. R. et al. Effects of thrombolysis during out-of-hospital cardiopulmonary resuscitation. Am. J. Cardiol. 2006; 97 (3): 305-308

25. Konstantinides S., Geibel A., Heusel G. et al. Heparin plus alteplase compared with heparin alone in patients with submassive pulmonary embolism. N. Engl. J. Med. 2002; 347 (15): 1143-1150.

26. Spohr F., Arntz H. R., Bluhmki E. et al. International multicentre trial protocol to assess the efficacy and safety of tenecteplase during cardiopulmonary resuscitation in patients with out-of-hospital cardiac arrest: The Thrombolysis in Cardiac Arrest (TROICA) Study. Eur. J.
Clin. Invest. 2005; 35 (5): 315-323.

27. Ames A., Wright R. L., Kowada M. et al. Cerebral ischemia II. The noreflow phenomenon. Am. J. Pathol. 1968; 52 (2): 437-453.

28. Ames A., Gurian B. Effects of glucose and oxygen deprivation on function of isolated mammalian retina. J. Neurophysiol. 1963; 26: 617-634.

29. Hirsch H., Euler K., Schneider M. Über die Erholung und Wiederbelebung des Gehirns nach Ischümie bei Normothermie. Pflügers Arch. Eur. J. Physiol. 1957; 265 (4): 281-313.

30. Rezkalla S., Kloner R. No-reflow phenomenon. Circulation 2002; 105 (5): 656-662.

31. Fischer E. G., Ames A. Studies on mechanisms of impairment of cerebral circulation following ischemia: effect of hemodilution and perfusion pressure. Stroke 1972; 3 (5): 538-542.

32. Fischer M., Hossmann K.-A. No-reflow after cardiac arrest. Intensive Care Med. 1995; 21 (2): 132-141.

33. Böttiger B. W., Krumnikl J.J., Gass P. et al. The cerebral no-reflow phenomenon after cardiac arrest in rats-influence of low-flow reperfusion. Resuscitation 1997; 34 (1): 79-87.

34. Hossmann $V$., Hossmann K.-A. Return of neuronal functions after prolonged cardiac arrest. Brain Res. 1973; 60 (2): 423-438.

35. Safar P., Abramson N. S., Angelos M. et al. Emergency cardiopulmonary bypass for resuscitation from prolonged cardiac arrest. Am. J. Emerg. Med. 1990; 8 (1): 55-67.

36. Iijima T., Bauer R., Hossmann K.-A. Brain resuscitation by extracorporeal circulation after prolonged cardiac arrest in cats. Intensive Care Med. 1993; 19 (2): 82-88.

37. Seo K., Ishimaru S., Hossmann K.-A. 2-stage resuscitation of the cat brain after prolonged cardiac arrest. Intens. Care Med. 1991; 17 (7): 410-418.

38. Hossmann K.-A., Kleihues P. Reversibility of ischemic brain damage. Arch. Neurol. 1973; 29 (6): 375-384.

39. Gando $S$., Kameue T. Nanzaki $S$. et al. Massive fibrin formation with consecutive impairment of fibrinolysis in patients with out-of-hospital cardiac arrest. Thromb. Haemost. 1997; 77 (2): 278-282

40. Gaszynski W. Research work on blood clotting system during cardiorespiratory resuscitation. Anaesth. Resusc. Intens. Ther. 1974; 2 (4): 303-316.

41. Anderson J. M., Brown J. K., Cockburn F. On the role of disseminated intravascular coagulation in the pathology of birth asphyxia. Dev. Med. Child Neurol. 1974; 16 (5): 581-591.

42. Wroblezeski M.,Jarzyna A., Obrzut A. The effect of cerebral anoxia on blood coagulation in the guinea-pig. Acta Neurochir. 1966; 15 (3): 263-268.

43. GeraudJ., Rascol A., Bierme R. et al. Troubles de la fonction plaquettaire et accidents ischemiques cerebraux. Perspectives therapeutiques. Rev. Neurol. 1972; 126 (1): 31-40.

44. Hossmann K.-A., Hossmann V. Coagulopathy following experimental cerebral ischemia. Stroke 1977; 8 (2): 249-254.

45. Lip G. Y., Blann A. D. Thrombogenesis and fibrinolysis in acute coronary syndromes. Important facets of a prothrombotic or hypercoagulable state? J. Am. Coll. Cardiol. 2000; 36 (7): 2044-2046.

46. Hossmann V., Hossmann K.-A., Takagi S. Effect of intravascular platelet aggregation on blood recirculation following prolonged ischemia of the cat brain. J. Neurol. 1980; 222 (3): 159-170.

47. Crowell J., Sharpe G., Lambright R. et al. The mechanism of death after resuscitation following acute circulatory failure. Surgery 1955; 38 (4): 696-702.

48. Crowell J. W., Smith E. E. Effect of fibrinolytic activation on survival and cerebral damage following periods of circulatory arrest. Am. J. Physiol. 1956; 186 (2): 283-285.

49. Lin S.-R., O'Connor M.J., Fischer H. W. et al. The effect of combined dextran and streptokinase on cerebral function and blood flow after cardiac arrest: an experimental study on the dog. Invest. Radiol. 1978; 13 (6): 490-498.

50. Sterz F., Safar P., Tisherman S. et al. Mild hypothermia cardiopulmonary resuscitation improves outcome after prolonged cardiac arrest in dogs. Crit. Care Med. 1991; 19 (3): 379-389

51. Hartveit F., Halleraker B. Intravascular changes in kidneys and lungs after external cardiac massage: a preliminary report. J. Pathol. 1970; 102 (1): 54-58.

52. Fischer M., Böttiger B. W., Popovcenic S. et al. Thrombolysis using plasminogen activator and heparin reduces cerebral no-reflow after resuscitation from cardiac arrest - an experimental study in the cat. Intensive Care Med. 1996; 22: 1214-1223.

53. Böttiger B. W., Padosch S. A. Thrombolysis using recombinant tissue-type plasminogen activator during cardiopulmonary resuscitation in patients with out-of- hospital cardiac arrest. Resuscitation 2002; 52 (3): 308-309.

54. Jan K., Powers E., Reinhart W. et al. Altered rheological properties of blood following administrations of tissue plasminogen activator and streptokinase in patients with acute myocardial infarction. Adv. Exp. Med. Biol. 1990; 281: 409-417.

Поступила 03.02.09 